\title{
A Study of Congestion Control Protocols for Wireless Sensor Networks
}

\author{
Harpreet Singh \\ M.Tech Research Scholar \\ Amritsar College of Engg. and Tech
}

\author{
Tanu Preet Singh, PhD \\ Professor and HOD \\ Amritsar College of Engg. and Tech
}

\begin{abstract}
Within the last couple of years a comparatively large amount of clustering routing protocols have already been developed for WSNs. This paper is an effort to comprehensively review and critically discuss the absolute most prominent clustering routing algorithms which have been developed for WSNs. The goals with this survey are to produce a large audience conscious of the existence and of the usually good performance of numerous clustering routing protocols in WSNs. From the survey, it has been discovered that none of technique performs effectively in most fields. Therefore the paper ends with the future scope to overcome these issues.
\end{abstract}

\section{Keywords}

WSNs, CLUSTERING PROTOCOLS, LEACH, PASCCC

\section{INTRODUCTION}

Clustering techniques in wireless sensor networks aims at gathering data among categories of nodes, which elect leaders among themselves. The leader or cluster-heads has got the role of aggregating the information and reporting the information to the BS. The advantages with this scheme is so it reduces energy usage of every node and communication cost. The clustering algorithms which are made is dependent on homogeneity and heterogeneity of nodes. Among the earliest work proposing this method in WSNs is LEACH. Recently, there have been a lot of other clustering techniques which are generally variants of LEACH protocol with slight improvement and different application scenarios. DEEC (Design of a distributed energy-efficient clustering), EDACH (Energy-Driven Adaptive Clustering Hierarchy) and EEUC (An EnergyEfficient Unequal Clustering Mechanism) are typical clustering techniques proposed with the goal of minimizing energy usage, while extending network life time. Clustered sensor network could be classified into two main types: homogeneous and heterogeneous sensor network. While energy efficiency in WSNs remains a function of uniform distribution of energy among sensor nodes, classifying clustering techniques is dependent upon the objectives in mind. The Optimal clustering technique may be the technique for the heterogeneity nodes.

\section{ADVANTGES AND OBJECTIVES OF CLUSTERING}

In contrast to flat routing protocols in WSNs, clustering routing protocols have a number of advantages, such as for instance more scalability, less load, less energy consumption and more robustness. In this section, we summarize these advantages in addition to the objectives of WSN clustering the following: More Scalability: In clustering routing scheme, sensor nodes are divided in too many different clusters with various assignment levels. The $\mathrm{CHs}$ are in charge of data aggregation, information dissemination and network management, and the MNs for events sensing and information collecting within their surroundings. Clustering topology can localize the route create within the cluster and thus reduce the size of the routing table stored at the individual sensor nodes. In contrast to a flat topology, this sort of network topology is simpler to handle, and more scalable to respond to events in the environment.

Data Aggregation/Fusion: Data aggregation/fusion, which can be the procedure of aggregating the information from multiple nodes to get rid of redundant transmission and provide fused data to the BS, is definitely an effectual technique for WSNs to save lots of energy. The most used data aggregation/fusion method is clustering data aggregation, where each $\mathrm{CH}$ aggregates the collected data and transmits the fused data to the BS. Usually $\mathrm{CHs}$ are formed a tree structure to transmit aggregated data by multi hopping through other $\mathrm{CHs}$ which results in significant energy savings.

Less Load: Since sensors might generate significant redundant data, data aggregation or fusion has emerged being an important tenet and objective in WSNs. The key concept of data aggregation or fusion is to mix data from different sources to get rid of redundant data transmissions, and provide an abundant and multi-dimensional view of the targets being monitored. Many clustering routing schemes with data aggregation capabilities require careful selection for clustering approach. For clustering topology, all cluster members only send data to $\mathrm{CHs}$, and data aggregation is conducted at the $\mathrm{CHs}$, that really help to dramatically reduce transmission data and save energy. Additionally, the routes are setup within the clusters which thus reduce the size of the routing table stored at the individual sensor nodes.

Less Energy Consumption: In clustering routing scheme, data aggregation really helps to dramatically reduce transmission data and save energy. Moreover, clustering with intra-cluster and inter-cluster communications can reduce the amount of sensor nodes performing the job of long-distance communications, thus allowing less energy consumption for the whole network. Additionally only $\mathrm{CHs}$ perform the job of data transmission in clustering routing scheme, which could save a lot of energy consumption. 
More Robustness: Clustering routing scheme causes it to be easier for network topology control and responding to network changes comprising node increasing, node mobility and unpredicted failures, etc. A clustering routing scheme only needs to manage with one of these changes within individual clusters, thus the whole network is better quality and easier for management. To be able to share the $\mathrm{CH}$ responsibility, $\mathrm{CHs}$ are usually rotated among most of the sensor nodes to prevent the single point of failure in clustering routing algorithms.

Collision Avoidance: In the multi-hop flat model, the wireless medium is shared and managed by individual nodes, thus this model can lead to low efficiency in the resource usage. On another hand, in the multi-hop clustering model, a WSN is divided in to clusters and data communications between sensor nodes comprise two modes, i.e., intra-cluster and inter-cluster, respectively for data collection and for data transmissions. Accordingly, resources could be allocated orthogonally to each cluster to lessen collisions between clusters and be reused cluster by cluster. Consequently, the multi-hop clustering model is suitable for large-scale WSNs.

Latency Reduction: Whenever a WSN is divided in to clusters, only $\mathrm{CHs}$ perform the job of data transmissions from the cluster. The mode of data transmissions only from the cluster helps avoiding collisions involving the nodes. Accordingly latency is reduced. Furthermore, data transmission is conducted hop by hop usually using the shape of flooding in flat routing scheme, but only $\mathrm{CHs}$ perform the job of data transmission in clustering routing scheme, which could decrease hops from data source to the BS, accordingly decrease latency.

Load Balancing: Load balancing is a vital consideration aiming at prolonging the network lifetime in WSNs. Even distribution of sensor nodes one of the clusters is generally considered for cluster construction where $\mathrm{CHs}$ perform the job of data processing and intra-cluster management. Generally constructing equal-sized clusters is adopted for prolonging the network lifetime because it prevents the premature energy exhaustion of CHs. Besides, multi-path routing is a technique to accomplish load balancing.

Fault-Tolerance: Because of the applicability of WSNs in an excellent many dynamic scenarios, sensor nodes may suffer with energy depletion, transmission errors, hardware malfunction, and malicious attacks and so on. With applications such as for instance hurricane modeling and tracking envisioned started using a large quantity of small sensor nodes, the cost of each sensor node is constrained. Owing to significant constraints on the cost, and therefore on the quality of sensor motes, and the often hostile environments by which they're deployed, sensor networks are vulnerable to failure. Thus, fault-tolerance is an essential challenge in WSNs. To be able to avoid the increasing loss of significant data from key sensor nodes, fault tolerance of $\mathrm{CHs}$ is generally required in this sort of applications, thus effective fault-tolerant approaches should be designed in WSNs. Re-clustering is the absolute most intuitive method to recover from a bunch failure, though it always disarranges the on-going operation. Assignment of $\mathrm{CH}$ backup is a practical scheme for recovery from the $\mathrm{CH}$ failure.
Guarantee of Connectivity: Sensor nodes usually transmit data to a number of BSs using a single-hop or multi-hop routing in WSNs, thus whether the information is successfully sent to the BS is principally determined by the connectivity of every node to its next hop node across the path. Furthermore, sensor nodes that cannot keep in touch with every other sensor node can get isolated and their data cannot be transmitted to the BS. Therefore, guarantee of connectivity is an important goal of clustering routing protocols in WSNs. An essential example is when some information concerning most of the sensor nodes must be collected by way of a designated fusion node in clustering routing protocols.

Energy Hole Avoidance: Generally, multi-hop routing can be used to provide the collected data to a drain or perhaps a BS. In those networks, the traffic transmitted by each node includes both self-generated and relayed traffic. Aside from MAC protocols, the sensor nodes nearer to the BS need certainly to transmit more packets than those far away from the BS. Consequently, the nodes nearer to the BS to deplete their energy first, leaving a gap close to the BS, partitioning the entire network, and avoiding the outside nodes from sending information to the BS, while many remaining nodes still have a lots of energy. This phenomenon is known as energy hole. Mechanisms of energy whole avoidance, i.e., energy consumption balancing, could be classified into three groups: node deployment, load balancing, along with energy mapping and assigning. Especially, uneven clustering is one of many ways of load balancing. In this process, an inferior cluster radius close to the sink and a more substantial cluster radius from the sink are defined respectively, so the power use of processing data in inter-cluster is less for cluster with smaller radius, and thus more energy may be used to relay data from remote nodes. On another hand, it's challenging to analyze the optimization of cluster radius theoretically.

Maximizing of the Network Lifetime: Network lifetime is definitely an inevitable consideration in WSNs, because sensor nodes are constrained in power, processing capability and transmission bandwidth, specifically for applications of harsh environments. Usually it's indispensable to minimize the power consumption for intra-cluster communication by $\mathrm{CHs}$ which are richer in resources than ONs. Besides, sensor nodes which can be near to all of the sensor nodes in the clusters must certainly be vulnerable to be CHs. Additionally, the goal of energyaware idea is to choose those routes which are likely to prolong the network lifetime in inter-cluster communications, and the routes made up of nodes with higher energy resources must certainly be preferred.

Quality of Service: The network applications and the functionalities of WSNs prompt the necessity of quality of service (QoS). Usually, effective sample, less delay and temporary precision are required. It's difficult for the routing protocols to satisfy all certain requirements of QoS, because some demands may breach a number of protocol principles. Existing clustering routing approaches in WSNs mainly concentrate on increasing energy efficient as opposed to QoS support. QoS metrics must be studied into consideration real-time applications, such as for instance battle-target tracking, emergent-event monitoring, and etc. 


\section{VARIOUS CLUSTERING PROTOCOLS}

\subsection{Low Energy Adaptive Clustering Hierarchy (LEACH)}

WSNs are micro sensor systems which are spatially distributed. WSN is just a power constrained system whilst the sensor nodes have limited battery life that shortens the network lifetime. Maximizing the network lifetime is determined by an efficient communication protocol. Energy consumption is, therefore, a crucial design issue in WSN. A cluster-based technique is the fundamental method to improve the scalability, performance, efficiency and duration of the network.

LEACH, a hierarchical clustering routing protocol, was proposed by Chandrakasan, Heinzelman and Balakrishnan, in MIT. Leach is really a protocol that is effectivein homogenous networks. In a homogenous network, all nodes have equal quantity of initial energy. Basically there are two kinds of routing protocols in WSNs: Flat routing protocols are those in which the routing condition of every node in the network may be the same. There are no special nodes in network and each node has equal status. So, the network traffic is distributed equally among all nodes. Comparatively, hierarchical routing protocols take advantage of the idea of clusters that divides all nodes into groups or clusters. Nodes in this kind of network have different levels. A CH is selected among all of the nodes and different hierarchical routing protocols may use different ways of selecting CHs.

LEACH is really a low energy protocol which could adapt clustering. It is just a cluster-based protocol that utilizes the idea of randomized rotation of local cluster-heads and distributes the power load evenly among most of the sensor nodes in the sensing field of the network.

\subsection{Stable Cluster Head Election (SCHE) Protocol}

It is dependent on LEACH architecture that uses clustering technique. Its goal is to lessen the power consumption of every sensor node and thus minimizing the entire energy dissipation of the network. SCHE is really a source driven protocol centered on timely reporting. Therefore the sensor node will always possess some data to transmit to the Base station. Additionally, it utilizes data aggregation to prevent information overload.

It gives an analytical framework to attain the stable probability for a node to be always a cluster-head to minimize energy consumption. It's necessary to use suitable $\mathrm{CH}$ election mechanism to minimize energy consumption of every sensor node that ultimately results in reduced energy dissipation. SCHE was proposed where this mechanism was applied by acquiring the optimum value of probability for a node becoming a $\mathrm{CH}$ and consumes considerably less energy in comparison to LEACH. In addition, it reduces consumption by minimizing distance between $\mathrm{CH}$ and BS.

\subsection{Stable Election Protocol (SEP)}

There are several drawbacks connected with LEACH such as for instance: single hop routing can be used where each node can transmit straight to $\mathrm{CH}$ and sink. $\mathrm{CHs}$ are elected randomly. Therefore there's possible that most $\mathrm{CHs}$ is likely to be concentrated in exactly the same area. The idea of dynamic clustering can be used that leads to unnecessary overhead as a result of cluster changes. The protocol also assumes that nodes have quantity of energy for every node.

But recent protocols like SEP have been opposite to that particular LEACH because it considers energy heterogeneity where in actuality the factors mentioned are simply a possibility. WSNs have assumed homogenous nodes for all of the time. But these nodes also differ in initial quantity of energy and also in depletion rate. This results in the heterogeneous networks where they considered several kinds of nodes. SEP is proposed for two-level heterogeneous networks that are two kinds of nodes according for their initial energy. The nodes which have higher quantity of energy compared to other nodes are called advance nodes and other nodes are the standard nodes.

In SEP the election probabilities of nodes are weighted by the original energy of each node to end up being the cluster-head in accordance with other nodes in a network. This prolongs the timeframe prior to the death of first node in the system. SEP approach makes sure $\mathrm{CH}$ election is performed randomly and is distributed on the basis of the energy of every node assuring the uniform usage of the nodes energy. SEP contains advance nodes that carry more energy compared to the normal nodes at the start so that it enhances the stability amount of the network.

\subsection{Extended Stable Election Protocol (ESEP)}

It's an altered SEP protocol. Rather than two kinds of nodes, it considers three nodes based on the energy levels. These nodes are: normal, moderate and advance nodes. The target of ESEP is to attain a WSN that maximizes the network lifetime and stability period. And yes it must reduce steadily the communication cost and deployment cost. The operation becoming a $\mathrm{CH}$ is just like in SEP by generating a random number and then comparing it with the threshold. In ESEP the moderate or intermediate nodes are selected in two ways either by the relative distance of advance nodes on track nodes or by the threshold of vitality between advance nodes and normal nodes.

The weighted election probabilities are given by:

$$
\begin{aligned}
p_{\text {nrm }}=\frac{p_{\text {opt }}}{1+p \cdot a+k \cdot b} & \\
p_{\text {mod }} & =\frac{p_{\text {opt }}}{1+p \cdot a+k \cdot b} *(1+\mathrm{a}) \\
p_{\text {adv }} & =\frac{p_{\text {opt }}}{1+p \cdot a+k \cdot b} *(1+\mathrm{b})
\end{aligned}
$$

And the total initial energy of heterogeneous network is given by:

$$
\begin{gathered}
E_{t}=n \cdot(1-p-k) \cdot E_{0}+n \cdot p \cdot E_{o} \cdot(1+\alpha) \\
+ \text { n.k. } E_{o}(1+b) \\
E_{t}=n \cdot(1-p \cdot a-k \cdot b) \cdot E_{0}
\end{gathered}
$$

The outcomes reveal that ESEP outperforms SEP and LEACH when it comes to stability due to three degrees of heterogeneity. However, $a$ additional energy factor between advance and normal nodes and $b$ additional energy factor between advance, normal and moderate nodes because of three kinds of nodes in ESEP, it's different energy levels. 


\subsection{Threshold-Sensitive Stable Election Protocol (TSEP)}

The first protocols SEP and ESEP were heterogeneityaware protocols that enhance the stability period and network lifetime but a significant drawback of heterogeneity is that the increased throughput eventually decreases the network lifetime. Therefore, to manage the trade-off involving the efficiency, accuracy and network lifetime, a new protocol TSEP was proposed. It is just a reactive routing protocol that senses data continuously within the network but transmits only when there is a drastic change in the worthiness of sensed attributes. The transmission happens only whenever a specific degree of threshold is reached. It uses three degrees of heterogeneity by considering three kinds of nodes: normal, intermediate and advance nodes. The greatest energy nodes are advance nodes followed closely by intermediate and normal nodes. The intermediate nodes are selected using a fraction $b$ of intermediate nodes. The power of intermediate nodes is assumed to be $\mu$ times significantly more than that of normal nodes

\subsection{Hierarchical Based Stable Election Protocol (HSEP)}

It's the following level protocol that has been proposed after ESEP. The increasing the distance involving the $\mathrm{CH}$ and the BS results in increasing the transmission energy because all of the energy is consumed in the transmission process. HSEP is proposed which aims at reducing the transmission energy involving the $\mathrm{CH}$ and BS. It brings into account the clustering hierarchy which lowers the transmission cost and hence the energy. In this kind of clustering used two kinds of cluster-heads: primary $\mathrm{CHs}$ and secondary $\mathrm{CHs}$.

The secondary $\mathrm{CHs}$ could be selected from the principal $\mathrm{CHs}$ and are elected on the foundation of probability from those nodes which had already end up being the primary $\mathrm{CHs}$. The principal $\mathrm{CHs}$ only can decide the secondary $\mathrm{CHs}$. They check distance between one another and those that are in minimum distance from their website are selected the secondary CHs. Additionally uses advance nodes and normal nodes. The procedure of selecting the principal $\mathrm{CH}$ is just like in Sep by generating a rando0m number between 0 and 1 and then comparing it with the threshold value. These primary $\mathrm{CHs}$ then aggregate data collected from other nodes and transmit it to the secondary $\mathrm{CHs}$ which further send it to the BS. Thus, minimizing the transmission distance involving the secondary $\mathrm{CHs}$ and the BS results in less use of energy.

Thus HSEP outperforms other protocols since it is dependent on clustering hierarchy by which $\mathrm{CHs}$ are of two levels. This hierarchical clustering reduces the transmission distance and hence results in less power dissipation. Also the stability amount of HSEP is higher when compared with others.

Thus, energy heterogeneity must certainly be among the key factors to be viewed when designing a strong protocol for WSN. The target is to create an altered protocol that's better quality and can ensure longer network lifetime while taking performance measures into consideration.

\section{PASCCC: PRIORITY-BASED APPLICATION-SPECIFIC CONGESTION CONTROL CLUSTERING PROTOCOL}

In this section, reveal description of distributed clusterbased routing protocol is given. To the most truly effective of knowledge, PASCCC is the initial protocol of its kind to consider mobility, heterogeneity, and congestion detection and mitigation utilizing a bunch hierarchy. Many studies have addressed congestion detection and mitigation, but they're either generic or specifically associated with the transport layer. Following assumptions in regards to the PASCCC are made:-

1. Nodes are deployed randomly in the field with an alternative number of energy values.

2. Nodes are designed for adjusting their transmission capacity to have the ability to reach an extremely distant $\mathrm{CH}$ on top of a specific round.

3. The positioning of a BS isn't fixed and it might be either within or away from sensor field.

4. Nodes are designed for moving throughout the field to cover vacant spaces utilizing the random waypoint mobility model with an interest rate $\mathrm{V}$, where the worthiness of $\mathrm{V}$ ranges between Vmin and Vmax. Hence, complete coverage of the sensor field is guaranteed.

In PASCCC, the nodes are designed for moving over the field if necessary to have the ability to cover vacant regions. Mobility ensures complete coverage and connectivity at all times. Hence, it's not as likely that a generated event is going unreported. In PASCCC, $10 \%$ of the nodes are advanced. These nodes have higher energy in contrast to normal nodes, thereby developing a heterogeneous amount of nodes in the network. PASCCC is definitely an application-specific protocol. In scheme, two application parameters are thought using PASCCC: temperature and humidity. PASCCC acts as a reactive protocol for temperature monitoring and as a proactive protocol for humidity. In reactive routing protocols, the nodes react immediately to sudden and drastic changes in the values of sensed events, and they're suitable for timecritical applications. In proactive routing protocols, the nodes switch on their transmitters, sense environmental surroundings, and report captured data periodically to the BS. These protocols are worthy of applications that need periodic data transmission.

\section{LITERATURE SURVEY}

Yi, Sangho et al. [1] proposed a PEACH protocol, which is really a power-efficient and adaptive clustering hierarchy protocol for wireless sensor networks. By utilizing overhearing characteristics of wireless communication, PEACH forms clusters without additional overhead and supports adaptive multi-level clustering. Additionally, PEACH may be used for both location-unaware and location-aware wireless sensor networks. The simulation results demonstrate that PEACH significantly minimizes energy consumption of every node and extends the network lifetime, in contrast to existing clustering protocols. The performance of PEACH is less suffering from the distribution of sensor nodes than other clustering protocols. Jiang, Chang-Jiang et al. [2] proposed an energy-balanced unequal clustering (EBUC) protocol. Utilizing the particle swarm optimization (PSO) algorithm, 
EBUC partitions all nodes into clusters of unequal size, in that the clusters nearer to the base station have smaller size. The cluster heads of those clusters can preserve even more energy for the inter-cluster relay traffic and the "hotspots 'problem could be avoided. For inter-cluster communication, EBUC adopts an energy-aware multihop routing to lessen the power use of the cluster heads. Simulation results demonstrate that the protocol can efficiently decrease the dead speed of the nodes and prolong the network lifetime. Bajaber, Fuad, and IrfanAwan [3] introduced an adaptive clustering protocol for wireless sensor networks, that is called Adaptive Decentralized Re-Clustering Protocol (ADRP) for Wireless Sensor Networks. In ADRP, the cluster heads and next heads are elected centered on residual energy of every node and the average energy of every cluster. The simulation results reveal that ADRP achieves longer lifetime and more data messages transmissions than current important clustering protocol in wireless sensor networks. Khalil, Enan A., and A. AtteaBara'a [4] reformulated the style of the most crucial characteristic of the EA in order to obtain a routing protocol that may provide better quality results than the present heuristic and meta-heuristic protocols when it comes to network stability period, lifetime, and energy consumption. An evolutionary-based routing protocol is proposed, which could guarantee better tradeoff involving the lifespan and the stability amount of the network with efficient energy utilization. To aid this claim, extensive simulations on 90 homogeneous and heterogeneous WSN models are evaluated and compared from the LEACH, SEP, and one of many existing evolutionary-based routing protocols, hierarchical clustering-algorithm-based genetic algorithm (HCR). Wang, Bang [5] provided a computation method for the perfect cluster size to minimize the average energy consumption rate per unit area. In the proposed coverageaware clustering protocol, they defined a cost metric that favors those nodes being more energy-redundantly covered as better candidates for cluster heads and select active nodes in ways that tries to emulate the absolute most efficient tessellation for area coverage. Their simulation results validate computation and show the significant improvement of the network coverage lifetime. Robert, Jean-Marc [6] proposed a novel clustering algorithm and a relay node selection algorithm on the basis of the residual vitality and connectivity index of the nodes. This hybrid model is described as $H$-OLSR. The OLSR messages are adapted to deal with the cluster heads election and the MPR nodes selection algorithms. These algorithms are made to cope with selfish nodes which are getting advantages of others without cooperating with them. Hence, they proposed an incentive compatible mechanism that motivates nodes to behave truthfully during the choice and election processes. Incentive retributions raise the trustworthiness of the nodes. Since network services are granted based on nodes' accumulated reputation, the nodes should cooperate. Finally, centered on nodes' reputation, the absolute most trusted forwarding paths are determined. This reputation-based hybrid model is known as RHOLSR. Simulation results reveal that the novel H-OLSR model centered on energy and connectivity can efficiently prolong the network lifetime, as the RH-OLSR model improves the trustworthiness of the network through the choice of the very trusted paths centered on nodes' reputations. They are both different processes used to define the reputation-based clustering OLSR (RBC-OLSR) routing protocol. Taheri, Hoda et al. [7] proposed an energy-aware distributed dynamic clustering protocol (ECPF) which applies three techniques: non-probabilistic cluster head $(\mathrm{CH})$ elections, fuzzy logic, and on demand clustering. The residual energy of the nodes is the principal parameter for electing tentative $\mathrm{CHs}$ using a nonprobabilistic fashion. A non-probabilistic $\mathrm{CH}$ election is implemented by introducing a delay inversely proportional to the rest of the energy of every node. Therefore, tentative $\mathrm{CHs}$ are selected based on the remaining energy. Additionally, fuzzy logic is employed to evaluate the fitness (cost) of a node to be able to choose a final $\mathrm{CH}$ from the group of neighboring tentative $\mathrm{CHs}$. On another hand, every regular (non $\mathrm{CH}$ ) node elects for connecting to the $\mathrm{CH}$ with the smallest amount of fuzzy cost in its neighborhood. Besides, in ECPF, $\mathrm{CH}$ elections are performed sporadically (in contrast to performing it every round). Simulation results demonstrate that their approach performs a lot better than well-known protocols (LEACH, HEED, and CHEF) when it comes to extending network lifetime and saving energy. Tyagi, Sudhanshu, and Neeraj Kumar [8] provided the taxonomy of numerous clustering and routing techniques in WSNs based on metrics such as for instance power management, energy management, network lifetime, optimal cluster head selection, multihop data transmission etc. An extensive discussion is provided in the text highlighting the relative advantages and disadvantages of most of the prominent proposals in this category which supports the designers to choose a specific proposal based on its merits within the others. Wang, Sheng-Shih, and Yi-Shiun Lin [9] proposed an inactive clustering aided routing protocol, named PassCAR, to improve routing performance in the one-way multi-lane highway scenario. The key goal of PassCAR is to find out suitable participants for constructing a wellbalanced and reliable cluster structure throughout the route discovery phase. Each candidate node self-determines its priority to compete for a participant utilizing the proposed multi-metric election strategy centered on metrics such as for instance node degree, expected transmission count, and link lifetime. Simulation results reveal that, in contrast to the initial PC mechanism, PassCAR not just advances the successful possibility of route discovery, but additionally selects more desirable nodes to take part in the created cluster structure. This well-constructed cluster structure significantly improves the packet delivery ratio and achieves an increased network throughput because of its preference for reliable, stable, and durable routing paths. $\mathrm{Gu}$, Xin et al. [10] proposed the ECDC (Energy and Coverage-aware Distributed Clustering Protocol), a builtin protocol involving both energy and coverage, which can be distinctive from previous clustering protocols. For different practical applications, they designed corresponding coverage importance metrics and introduce them in to the clustering algorithm. Theoretical analysis and simulation results reveal that their protocol works well in improving network coverage performance, reducing nodes energy dissipation and extending the network lifetime. Akkari, Nadine et al. [11] proposed a new dynamic protocol for clustering the nodes considering the possible changes occurring in a cellular network. Specifically, the Dynamic Clustering Protocol (DCP) adapts the network configuration with the variable mobiles' requirements and the various network events. This can reduce steadily the needed time and signaling and offers better service quality for the clustered users. After presenting the different network events, the handover scenarios and signaling for the Dynamic 
Clustering Protocol, the performance of the proposed protocol is studied. This is accomplished by modeling different network scenarios and computing the necessary quantity of handovers as a function of user mobility, available network resources and data rate requirements for confirmed clustered nodes configuration. Xia, Feng et al. [12] proposed a new clustering protocol, namely BeeCup, to truly save the power of cellular devices while guaranteeing the quality of learning. The BeeCup protocol takes benefit of biologically-inspired computation, with concentrate on improving the power efficiency of mobile devices. It first estimates how many cluster heads $(\mathrm{CHs})$ adaptively based on the network scale, and then selects the $\mathrm{CHs}$ by employing the $\mathrm{ABC}$ algorithm. Just in case some $\mathrm{CHs}$ consume energy excessively, clusters will soon be dynamically updated to help keep energy consumption balanced within the complete network. Simulation results demonstrated the effectiveness and superiority of the proposed protocol Mirsadeghi, Maryam et al. [13] proposed a new distributed clustering approach using fuzzy logic. It uses Fuzzy logic to assign $\mathrm{CH}$ selection opportunity to network nodes to be able to choose tentative and final $\mathrm{CHs}$. simulation results demonstrate that their approach reachs to higher network lifetime, energy efficiency and network coverage when comparing to UCFIA, GCA, and SCP. Jan, Mian Ahmad et al. [14] proposed a priority-based application-specific congestion control clustering (PASCCC) protocol, which integrates the mobility and heterogeneity of the nodes to detect congestion in a network. PASCCC decreases the work cycle of every node by maintaining threshold levels for various applications. The transmitter of a sensor node is triggered once the reading of a particular captured event exceeds a particular threshold level. Time-critical packets are prioritized during congestion to be able to maintain their timeliness requirements. Within their proposed approach, $\mathrm{CHs}$ ensure coverage fidelity by prioritizing the packets of distant nodes over those of nearby nodes. A novel queue scheduling mechanism is proposed for $\mathrm{CHs}$ to accomplish coverage fidelity, which ensures that the additional resources consumed by distant nodes are utilized effectively. The potency of PASCCC was evaluated centered on comparisons with existing clustering protocols. The experimental results demonstrated that PASCCC achieved better performance when it comes to the network lifetime, energy consumption, data transmission, and other QoS metrics in contrast to existing approaches. Elhabyan, Riham SY, and Mustapha CE Yagoub [15] presented two Linear Programming (LP) formulations to the issues of clustering and routing followed closely by two proposed algorithms for exactly the same centered on Particle Swarm Optimization (PSO). The clustering algorithm finds the perfect group of $\mathrm{CHs}$ that maximize the energy efficiency, cluster quality and network coverage. The routing algorithm is developed with a novel particle encoding scheme and fitness function to obtain the optimal routing tree that connects these $\mathrm{CHs}$ to the BS. Those two algorithms are then combined right into a two-tier protocol to supply an entire and practical clustering model. The aftereffect of employing a realistic network and energy consumption model in cluster-based communication for WSN is likely to be investigated. Extensive simulations on 50 homogeneous and heterogeneous WSN models are evaluated and compared against well-known cluster-based sensor network protocols. The outcomes demonstrate that the proposed protocol performs much better than such protocols when it comes to various performance metrics such as for instance scalability, Packet Delivery Rate (PDR) at the $\mathrm{CHs}$ and delivery of total data packets to the BS. Sert, Seyyit et al. [16] introduced a new clustering approach that will be not just energy-efficient but additionally distributionindependent for wireless sensor networks (WSNs). Clustering can be used as a method of efficient data gathering technique with regards to energy consumption. In clustered networks, each node transmits acquired data to a cluster-head which the nodes belong to. Following a cluster-head collects all the information from all member nodes, it transmits the information to the base station (sink) either in a compressed or uncompressed manner. This data transmission occurs via other cluster-heads in a multi-hop network environment. Consequently of this case, cluster-heads near to the sink have a tendency to die earlier due to the heavy inter-cluster relay. This issue is named because the hotspots problem. To resolve this issue, some unequal clustering approaches have previously been introduced in the literature. Unequal clustering techniques generate clusters in smaller sizes when approaching the sink to be able to decrease intra-cluster relay. Along with the hotspots problem, the energy hole problem might also occur due to the changes in the node deployment locations. Although numerous previous studies have centered on energy-efficiency in clustering, to the very best of knowledge, none considers both problems in uniformly and non-uniformly distributed networks. Therefore, they proposed a multi-objective solution for these problems. In this study, they introduced a multi-objective fuzzy clustering algorithm (MOFCA) that addresses both hotspots and energy hole problems in stationary and evolving networks. Performance analysis and evaluations are completed with popular clustering algorithms and obtained experimental results reveal that MOFCA outperforms the present algorithms in exactly the same setup when it comes to efficiency metrics, which are First Node Dies (FND), half the Nodes Alive (HNA), and Total Remaining Energy (TRE) employed for estimating the duration of the WSNs and efficiency of protocols. Peng, Shuai et al. [17] proposed distributive Energy Neutral Clustering (ENC) protocol to group the network into several clusters, with the target of providing perpetual network operation. ENC employs a novel Cluster Head Group (CHG) mechanism which allows a cluster to utilize multiple cluster heads to generally share heavy traffic load. This CHG mechanism will help reduce steadily the frequency of cluster re-formations, which reduces the control message overhead. The optimum quantity of clusters that maximizes the quantity of information gathered from the network is mathematically derived using convex optimization techniques. Based with this optimum quantity of clusters, an expansion to ENC is proposed to group the network into equal sized clusters to ensure that maximized network information gathering may be achieved. Extensive empirical studies reveal that their proposed protocol can successfully prevent sensors from turning off because of the excessive usage of energy, which provides perpetual network operation with consistent data delivery. Substantial improvements on the quantity of information gathered from the network may also be attained by utilizing their proposed protocol when compared with traditional clustering protocols. Zhu, Jiang et al. [18] proposed a hybrid clustering protocol - Hybrid Distributed Hierarchical Agglomerative Clustering ( $\mathrm{H}-$ DHAC) - which uses both quantitative location data and binary qualitative connectivity data in clustering for 
WSNs. Their simulation results demonstrate that H-DHAC only includes a slightly lower percentage of compromise in performance when it comes to network lifetime and total transmitted data in comparison to similar approaches that use complete location data. However, H-DHAC still outperforms the well-known clustering protocols, e.g., LEACH and LEACH-C. On another hand, the cost of $\mathrm{H}-$ DHAC could be significantly lower compared to those approaches that use complete quantitative location data, as GPS isn't required for several sensor nodes. Additionally, H-DHAC still could be operational in the clear presence of GPS failures. Gielow, Fernando et al. [19] proposed architecture for dynamic and distributed data-aware clustering, and the Dynamic Data-aware Firefly-based Clustering (DDFC) protocol to deal with spatial similarity between node readings. The DDFC operation takes into consideration the biological principles of fireflies to make sure distributed synchronization of the clusters' similar readings aggregations. DDFC was in comparison to other protocols and the outcomes demonstrated its convenience of maintaining synchronized cluster readings aggregations, thereby enabling nodes to be dynamically clustered according with their readings.

\section{CONCLUSION AND FUTURE SCOPE}

In this study, we present an extensive survey of different clustering routing protocols proposed in recent years. Clustering is a great technique to lessen energy consumption and to supply stability in wireless sensor networks. From the survey it's been figured none of the technique performs effectively in most fields. Therefore in for seeable future, improvement can be carried out to boost the results.

\section{REFERENCES}

[1] Yi, Sangho, JunyoungHeo, Yookun Cho, and Jiman Hong. "PEACH: Power-efficient and adaptive clustering hierarchy protocol for wireless sensor networks." Computer communications 30 , no. 14 (2007): 2842-2852.

[2] Jiang, Chang-Jiang, Wei-Ren Shi, and Xian-lun TANG. "Energy-balanced unequal clustering protocol for wireless sensor networks." The Journal of China Universities of Posts and Telecommunications 17, no. 4 (2010): 94-99.

[3] Bajaber, Fuad, and IrfanAwan. "Adaptive decentralized re-clustering protocol for wireless sensor networks." Journal of Computer and System Sciences 77, no. 2 (2011): 282-292.

[4] Khalil, Enan A., and A. AtteaBara'a. "Energy-aware evolutionary routing protocol for dynamic clustering of wireless sensor networks." Swarm and Evolutionary Computation 1, no. 4 (2011): 195-203.

[5] Wang, Bang, Hock Beng Lim, and Di Ma. "A coverage-aware clustering protocol for wireless sensor networks." Computer Networks 56, no. 5 (2012): 1599-1611.

[6] Robert, Jean-Marc, HadiOtrok, and AbdelkarimChriqi. "RBC-OLSR: Reputation-based clustering OLSR protocol for wireless ad hoc networks."Computer Communications 35, no. 4 (2012): 487-499.
[7] Taheri, Hoda, PeymanNeamatollahi, Ossama Mohamed Younis, ShahrzadNaghibzadeh, and Mohammad Hossein Yaghmaee. "An energy-aware distributed clustering protocol in wireless sensor networks using fuzzy logic." Ad Hoc Networks 10, no. 7 (2012): 1469-1481.

[8] Tyagi, Sudhanshu, and Neeraj Kumar. "A systematic review on clustering and routing techniques based upon LEACH protocol for wireless sensor networks."Journal of Network and Computer Applications 36, no. 2 (2013): 623-645.

[9] Wang, Sheng-Shih, and Yi-Shiun Lin. "PassCAR: A passive clustering aided routing protocol for vehicular ad hoc networks." Computer Communications 36, no. 2 (2013): 170-179.

[10] Gu, Xin, Jiguo Yu, Dongxiao Yu, Guanghui Wang, and Yuhua Lv. "ECDC: An energy and coverageaware distributed clustering protocol for wireless sensor networks." Computers \& Electrical Engineering 40, no. 2 (2014): 384-398.

[11] Akkari, Nadine, GhadahAldabbagh, Michel Nahas, and John Cioffi. "Dynamic Clustering Protocol for coordinated tethering over cellular networks." Journal of Network and Computer Applications 42 (2014): 92101.

[12] Xia, Feng, Xuhai Zhao, Jianhui Zhang, Jianhua Ma, and Xiangjie Kong. "BeeCup: A bio-inspired energyefficient clustering protocol for mobile learning."Future Generation Computer Systems 37 (2014): 449-460.

[13] Mirsadeghi, Maryam, Ali Mahani, and Maryam Shojaee. "A Novel distributed Clustering Protocol using fuzzy logic." Procedia Technology 17 (2014): 742-748.

[14] Jan, Mian Ahmad, Priyadarsi Nanda, Xiangjian He, and Ren Ping Liu. "PASCCC: Priority-based application-specific congestion control clustering protocol." Computer Networks 74 (2014): 92-102.

[15] Elhabyan, Riham SY, and Mustapha CE Yagoub. "Two-tier particle swarm optimization protocol for clustering and routing in wireless sensor network."Journal of Network and Computer Applications 52 (2015): 116-128.

[16] Sert, SeyyitAlper, HakanBagci, and Adnan Yazici. "MOFCA: Multi-objective fuzzy clustering algorithm for wireless sensor networks." Applied Soft Computing 30 (2015): 151-165.

[17] Peng, Shuai, T. Wang, and C. P. Low. "Energy Neutral Clustering for Energy Harvesting Wireless Sensors Networks." Ad Hoc Networks (2015).

[18] Zhu, Jiang, Chung-Horng Lung, and VineetSrivastava. "A hybrid clustering technique using quantitative and qualitative data for wireless sensor networks."Ad Hoc Networks 25 (2015): 38-53.

[19] Gielow, Fernando, Gentian Jakllari, Michele Nogueira, and Aldri Santos. "Data similarity aware dynamic node clustering in wireless sensor networks." Ad Hoc Networks 24 (2015): 29-45. 